\title{
A real-time platform for monitoring schistosomiasis transmission supported by Google Earth and a web-based geographical information system
}

\author{
Kun Yang ${ }^{1,2}$, Le-Ping Sun ${ }^{1,2}$, Yi-Xin Huang ${ }^{1,2}$, Guo-Jing Yang ${ }^{1,2}$, Feng Wu ${ }^{1,2}$, De-Rong Hang ${ }^{1,2}$, \\ Wei $\mathrm{Li}^{1,2}$, Jian-Feng Zhang ${ }^{1,2}$, Yong-Sheng Liang ${ }^{1,2}$, Xiao-Nong Zhou ${ }^{3,4}$ \\ ${ }^{1}$ Jiangsu Institute of Parasitic Diseases, Wuxi 214064, People's Republic of China; ${ }^{2}$ Key Laboratory on \\ Technology for Parasitic Disease Prevention and Control, Ministry of Health, Wuxi 214064, People's Republic \\ of China; ${ }^{3}$ National Institute of Parasitic Diseases, Chinese Center for Disease Control and Prevention, \\ Shanghai 200025, People's Republic of China; ${ }^{4}$ WHO Collaborating Center for Malaria, Schistosomiasis and \\ Filariasis, Key Laboratory of Parasite and Vector Biology, Ministry of Health, Shanghai, 200025, People's \\ Republic of China
}

\begin{abstract}
A basic framework for the rapid assessment of the risk for schistosomiasis was developed by combining spatial data from Google Earth ${ }^{\circledR}$ with a geographical information system (GIS) package, bundling the modules together with an Internet connection into a WebGIS platform. It operates through functions such as "search", "evaluation", "risk analysis" and "prediction" and is primarily aimed to be a dynamic, early-warning system (EWS) providing user-friendly, evidencebased, near real-time awareness of the status of an important endemic disease. It contributes to rapid information-sharing at all levels of decision-making, facilitating "point-of-care" response, i.e. treatment provided at newly discovered transmission sites. The experience using the platform is encouraging and it has the potential to improve support systems and strengthen schistosomiasis control activities, in particular with regard to surveillance and EWS. It can quickly and intuitively locate early, high-risk areas, retrieve all important data needed as well as provide detailed, up-to-date information on the performance of the control programme. This WebGIS, the first of its kind in the People's Republic of China, is not only applicable for schistosomiasis but can easily be adapted for improving control of any endemic disease in any geographical area.
\end{abstract}

Keywords: geographical information system, remote sensing, spatio-temporal epidemiology, Google Earth, real-time surveillance, early-warning system, schistosomiasis, People's Republic of China.

\section{Introduction}

Remotely sensed data on land cover, elevation, temperature, rainfall, etc. complement geographical information systems (GIS) by providing access to continuously updated background information. The Internet has played an integral part in this development allowing scientists, control officers and decision-makers shared access to near real-time data. The presentation of data based on interactive, computer-generated map applications represents a straightforward way of visualizing large numbers of datasets in a geographical context for the non-specialist.

\footnotetext{
Corresponding author:

Xiao-Nong Zhou

National Institute of Parasitic Diseases

Chinese Center Diseases Control and Prevention

Shanghai 200025

People's Republic of China

Tel. +8621 64738058; Fax +862164332670

E-mail address: ipdzhouxn@sh163.net
}

These tools have proved particularly useful for the study of infectious agents relying on intermediate hosts for the completion of their lifecycles, since both are vulnerable to changes in temperature, humidity, and vegetation cover. The improved possibilities to study the boundaries of the intermediate hosts and vectors, on which parasites often depend, have promoted the field for gaining epidemiological information from satellite imagery (Hendrickx et al., 2004; Cringoli et al., 2005; Rinaldi et al., 2006). In addition, remote sensing had a strong impact on the creation of virtual globes with far-reaching consequences for any activities having to do with map-based information. The industry has not been slow in utilizing this novel way of collating and visualizing this information (http://www.google.com/earth/index.html), nor have professional epidemiologists deferred using this novel approach (Stensgaard et al., 2009).

Many endemic diseases can be expected to occupy new areas when climatic conditions change. This scenario looks increasingly likely, for example with 
respect to schistosomiasis in the People's Republic of China (P.R. China) (Yang et al., 2006) and leishmaniasis and dirofilariasis in Italy (Otranto et al., 2009). Indeed, several vector-borne diseases have emerged and spread due to global and/or local changes (Hendrickx et al., 2004; Rogers and Randolph, 2006). The current increase in international trade, tourism and travel are also important drivers for the proliferation of vector-borne parasites into new geographical areas (Harrus and Baneth, 2005).

The parasitic trematode worm Schistosoma japonicum, the cause of schistosomiasis in P.R. China, requires the presence of a snail intermediate host, Oncomelania hupensis, to complete its lifecycle. This snail is common in the streams, great lakes and along the Yangtze River in the southern part of the country. Strong advances in the control of S. japonicum have been made in P.R. China, but elimination of schistosomiasis has yet to be achieved (Zhou et al., 2005, 2007). Chinese applications of remote sensing and GIS for the study of the epidemiology of Schistosomiasis have shown potential for improving the spatio-temporal aspects of disease surveillance including the development of risk-mapping and predictive distribution studies at scales ranging from micro to macro. Prediction maps have been generated by the Bayesian Poisson spatial model together with environmental surrogates and landscape metrics to predict the spatial distribution of O. hupensis locally (Yang et al., 2005a, 2008, 2009; Zhou et al. 2009, 2010). However, the approach is far from straightforward and there are obstacles restricting full utilization of the new technologies. Firstly, spatio-temporal epidemiology is a sophisticated profession requiring training, expertise and experience. Secondly, the cost of the necessary, specialized software such as ArcGIS (http://www.esri.com/software/arcgis/index.html), MapInfo (http://www.pbinsight.se/) or ERDAS (http://www.erdas.com/Homepage.aspx) is comparatively high since the software comes in packages containing a multitude of functions, only a few of which are required in epidemiological research. Thirdly, realtime monitoring (e.g. of risk factors) is a necessity for early warning systems (EWS), without which counter responses would have a much reduced effect.

GIS applications, although sometimes costly, are acquired at an ever increasing pace, which is likely to result in an exponential increase of the number of potential users (Kamel Boulos, 2004; Chang et al., 2009). The impediments related to cost and distribution of findings can be overcome by the current availability of free software for mapping and the use of the
Internet for dissemination of the information (Kamel Boulos, 2004; Ptochos et al., 2004; Kamadjeu and Tolentino, 2006; Zhou et al., 2009). In addition, Google Earth ${ }^{\circledR}$ provides free access to satellite imagery along with editing tools for the generation of data-layers for terrain, boundaries and socioeconomic infrastructures (Conroy et al., 2008; Lefer et al., 2008; Lozano-Fuentes et al., 2008; Chang et al., 2009). The aim of this study is to describe the design and testing of a real-time, web-based platform (WebGIS) for schistosomiasis surveillance in P.R. China.

\section{Materials and methods}

\section{Study area}

The lower reaches of the Yangtze River in Jiangsu province, covering $102,600 \mathrm{~km}^{2}$ along the eastern coast of P.R. China with a population of about 72 million, was chosen as trial area for the proposed approach to schistosomiasis control. Even if the situation has improved along with the progress of the schistosomiasis control programme, this area still contained $72 \mathrm{~km}^{2}$ of snail habitats and $18 \mathrm{~km}^{2}$ of highrisk areas in 2006 (Sun et al., 2008; Hong et al., 2011). It can therefore be expected that thousands of people still run the risk of becoming infected (or reinfected) with $S$. japonicum each year.

\section{Development of the database}

Designed to encompass broad information on geographical risk, the database is divided into three components: one for the prevalence of the parasite in its definite hosts (humans and bovines for now, but keeping the door open for the inclusion of other species in the future), one for the snail intermediate host, its distribution, infection prevalence and presence of cercariae (the infective stage of the parasite) in the water, and one for the transmission risk owing to the characteristics of general environmental. Risk maps were created by merging information on schistosomiasis epidemiology and cercarial presence in water-bodies along with landscape features of epidemiological interest. This information depends on processing and storage of original data in the three components of the database. The server side of the database is interactive in the sense that, in addition to downloading disease-related information, the user may also upload data to be shared with others.

The database was fed with data from previous field surveys for schistosomiasis in humans and bovines as 
well as data on snail densities in various places, including their infection rates. These data are continuously updated by ongoing field surveys, with the coordinates of collection sites indicated by global positioning systems (GPS) as suggested by previous research (Zhou et al., 2001; Boulos, 2005; Yang et al., 2008, 2009; Sun et al., 2009). A typical field survey would screen about $90 \%$ of all residents in an area by serology, focusing on individuals aged 6-60 years. Commonly used assays for $S$. japonicum antibodies are the indirect hemagglutination assay (IHA) (Jia et al., 2009) or the dipstick dye immunoassay (DDIA) developed by Zhu et al. (2005). Seropositive individuals would be followed up by stool examination using triplicate Kato-Katz thick smears from each sample (Zhou et al., 2008b). The input for the database area dealing with cercarial presence at selected sites is based on previous work by Spear et al. (2004) and Sun et al. (2011) arranging wire cages containing sentinel mice above the water but making sure that their tails, paws, and portions of the lower abdomen have water contact. After exposure for two consecutive days between 10:00 and 15:00 hours (the time cercarial release from the infected snail reaches its peak), the mice were kept for 5 weeks to allow maturation of any cercariae into adult worms in vivo. The mice would subsequently be killed, dissected, any discovered worms counted, and the results imported into the database to be used as a local risk indictor.

While the first two datasets provide exact measures (prevalence in definite hosts, snail distributions and cercarial presence), the third dataset was designed to reflect various degrees of risk for infection in the general environment such as the vegetation index, land cover, snail habitats as well as the locations of water- bodies, villages, pastures, etc. Since KML (Keyhole Markup Language), a standard format for geographic map annotations in Google Earth ${ }^{\circledR}$, is commonly used to import this kind of information as shapefiles for advanced spatial analysis and modelling in combination with remote sensing images, we selected the intergrated Google Earth ${ }^{\circledR}$ technolgy for the development of the WebGIS platform. The infrastructure data layers were produced showing marshlands as labelled polygons, rivers, ditches and streams as lines, and villages and other sites of interest as points. Google Earth $^{\circledR}$ was also used to produce general-purpose static maps.

\section{Platform architecture}

A database service built on server technology was selected making sure that the information stored would be easily available and automatically run with a minimum of administration. We used the architecture for the platform described before (Yang et al., $2005 \mathrm{~b}$ ) as it meets the requirements mentioned above and is also secure, light-weight and simple to customize. It consists of a server side with the database containing the three types of data described above as the first tier, the components related to processing (web server, map server and user interface) as the second, while the third tier comprises the client side (Fig. 1). Before displaying the interface, the map server retrieves the spatial data from the database.

The software environment of this platform relies on various open standards including the Hypertext Markup Language (HTML) and the Geography Markup Language (GML) for the expression of geographical features. HTML was used for development

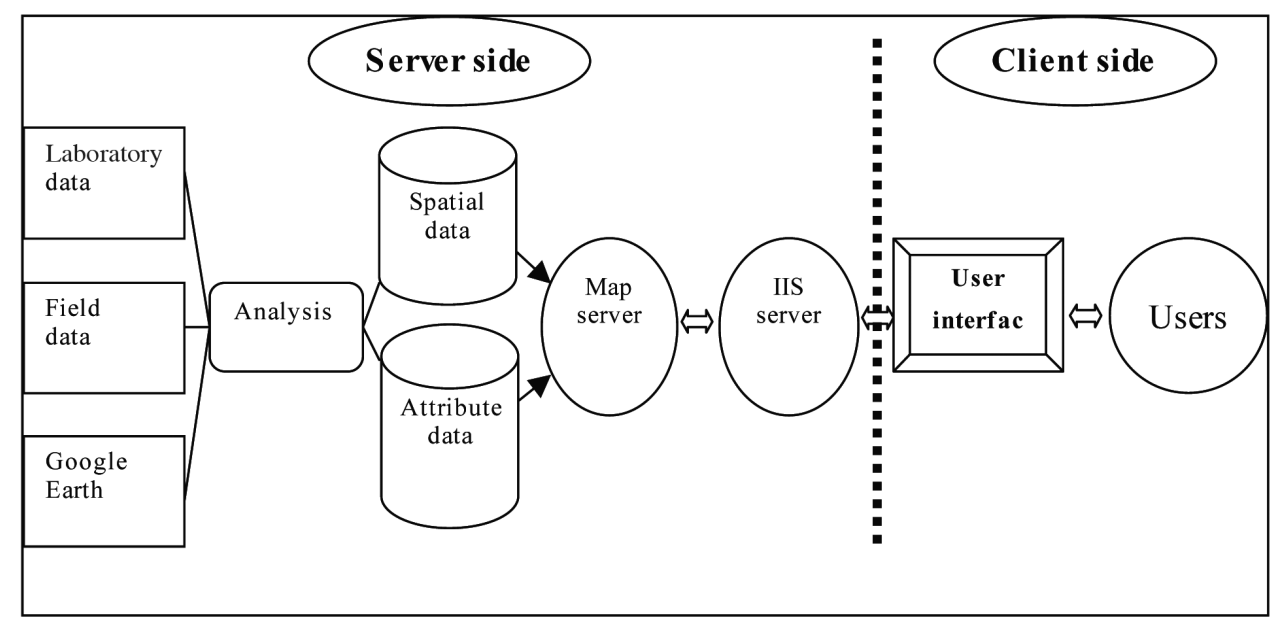

Fig. 1. Schematic overview of the architecture of the real-time, web-based, monitoring platform for schistosomiasis control. 
of the Webpage interface, and GML for the transfer of the commands between server and the client side.

\section{The server side}

The Microsoft SQL Server 2000 (http://www. microsoft.com/sqlserver/en/us/default.aspx) was used for merging the database contents with the mapping capabilities, which relies on the Arc Internet Map Server (ArcIMS) produced by ESRI (Redlands, CA, USA) for interactive mapping. The database, based on information from field surveys, laboratory tests and spatial information, includes three main types of data discussed above. The WebGIS, containing a number of annotated maps to be used as overlays, can be operated from any basic computer with Internet connection. This approach was developed by converting the spatial data from the SQL server into eXtensible Markup Language (XML)-formatted coordinates using ArcSDE, a server-software sub-system enabling the presentation of the information as a geodatabase. An ArcIMS Servlet connector was used to securely define the Hypertext Transfer Protocol (HTTP) classes. This type of connector functions as a protected link, receiving the requests via the web server and passing them on to the application server in ArcXML format. This combination of the spatial framework with the data repository allows the storage of GIS data files in a central location for easy access and management, while maintaining a tight linkage to the master database.

\section{The client side}

The principal function of the client side is to monitor and share all information stored in the server side of the database in real time. In addition, these data are interactive in the sense that the user can also upload information to be shared with others. The platform has a graphical user interface enabling public health officers, managers and policymakers at all levels, including staff at the headquarters at the Ministry of Health $(\mathrm{MoH})$, to work together. The user-friendly interface and the interactive mode of operation are essential components facilitating convenient and flexible exchange of information. The web browser provides the client with information on the geographic distribution of schistosomiasis (a function that can be extended to include any epidemic disease) when requested. This function is dynamic, i.e. it permits zooming, panning, production of theme maps, identification of risk areas and prediction of risk zones. It can be said to be fully interactive since the user can select and evaluate environmental and social attribute data, analyse spatio-temporal trends, display and define circles of different diameters around points of interest. Naturally, the client can also be utilized to alert local health officers of possible outbreaks, assess resource allocation and participate in decision-making activities supporting prevention and control.

\section{Real-time surveillance}

For monitoring purposes, spatio-temporal models are currently used for the prediction of snail habitats and S. japonicum prevalence, relying on spatial statistics to confirm high-risk areas, identified and located utilizing specific ArcGIS tools such as the "Spatial Analyst” (http://www.esri.com/software/arcgis/extensions/spatialanalyst/index.html), the "Geostatistical Analyst" (http://www.esri.com/software/arcgis/extensions/geostatistical/index.html) or $\mathrm{R}$ software (http: //www.r-project.org/).

Risk management is based on data obtained from various Earth-observing satellites, the capabilities of which have been tabulated by various authors (Lillesand and Kiefer, 1987; Bergquist, 2011), selecting environmental parameters of importance for the distribution of O. hupensis, i.e. soil, elevation, vegetation and hydrology. As recommended by previous studies (Yang et al., 2005a, 2008; Zhou et al., 2008a, 2010; Malone et al., 2010), risk prediction is performed based on a model using the following georeferenced data-layers:

(i) epidemiological records from the $1980 \mathrm{~s}$ to the 2010s for the lower Yangtze River basin with emphasis on parameters affecting snail habitats and disease prevalence (e.g. flooding patterns and environmental factors);

(ii) GIS layers based on remotely sensed data focused on seasonal changes of snail habitats and active transmission sites; and

(iii) spatio-temporal outcomes of the mice bioassay described above.

\section{Implementation}

Figures 2 and 3 show the client side interface of the platform, which is currently running on the local area network of Jiangsu Institute of Parasitic Diseases (JIPD), situated in Wuxi, Jiangsu province north of Shanghai. The real-time monitoring includes the analysis of GIS and remote sensing data as well as visualization of the distributions of schistosomiasis and snail habitats. The latter information is not only 


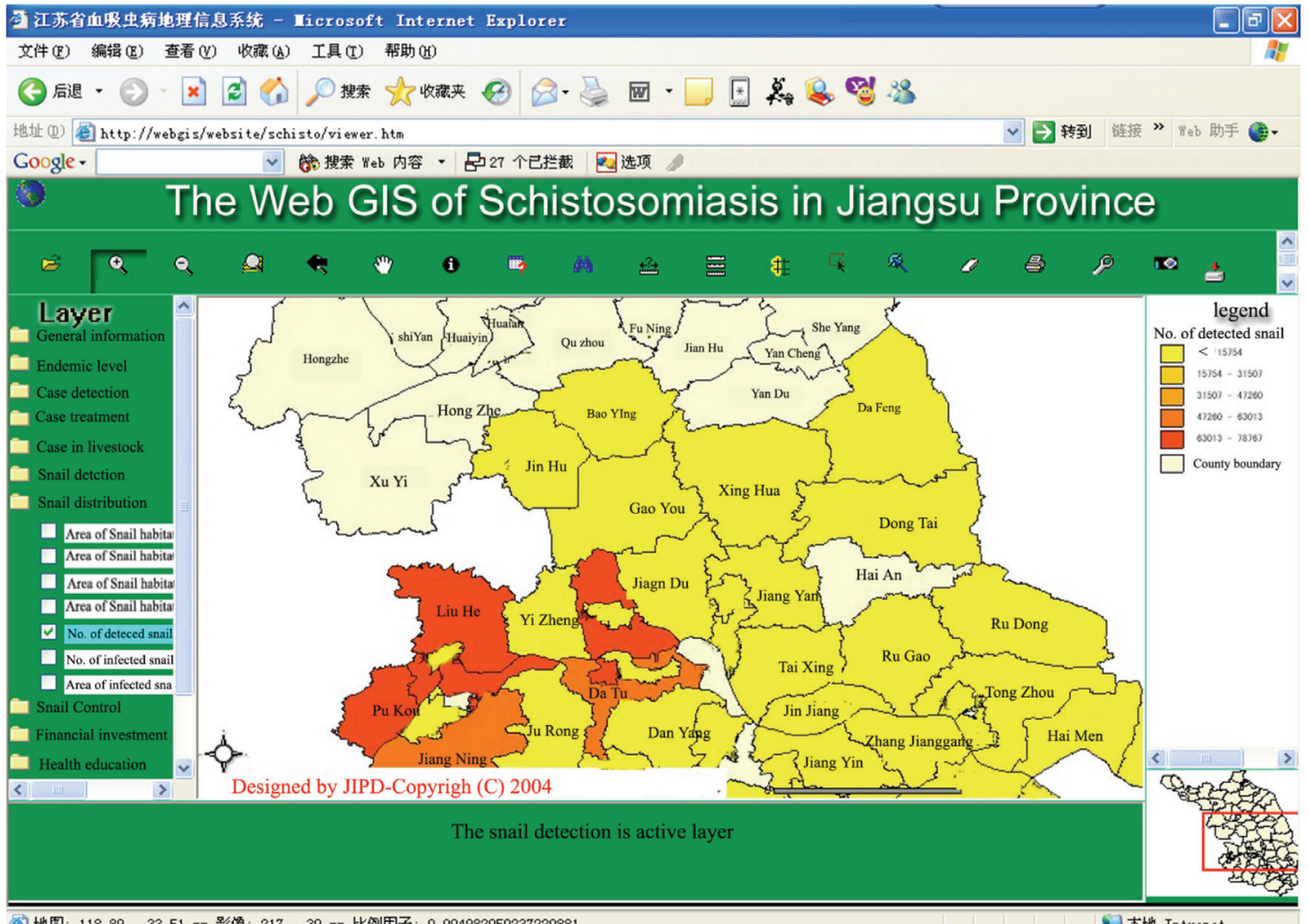

Fig. 2. The interface of the real-time platform for monitoring schistosomiasis transmission in Jiangsu province, China.

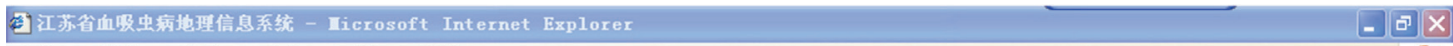

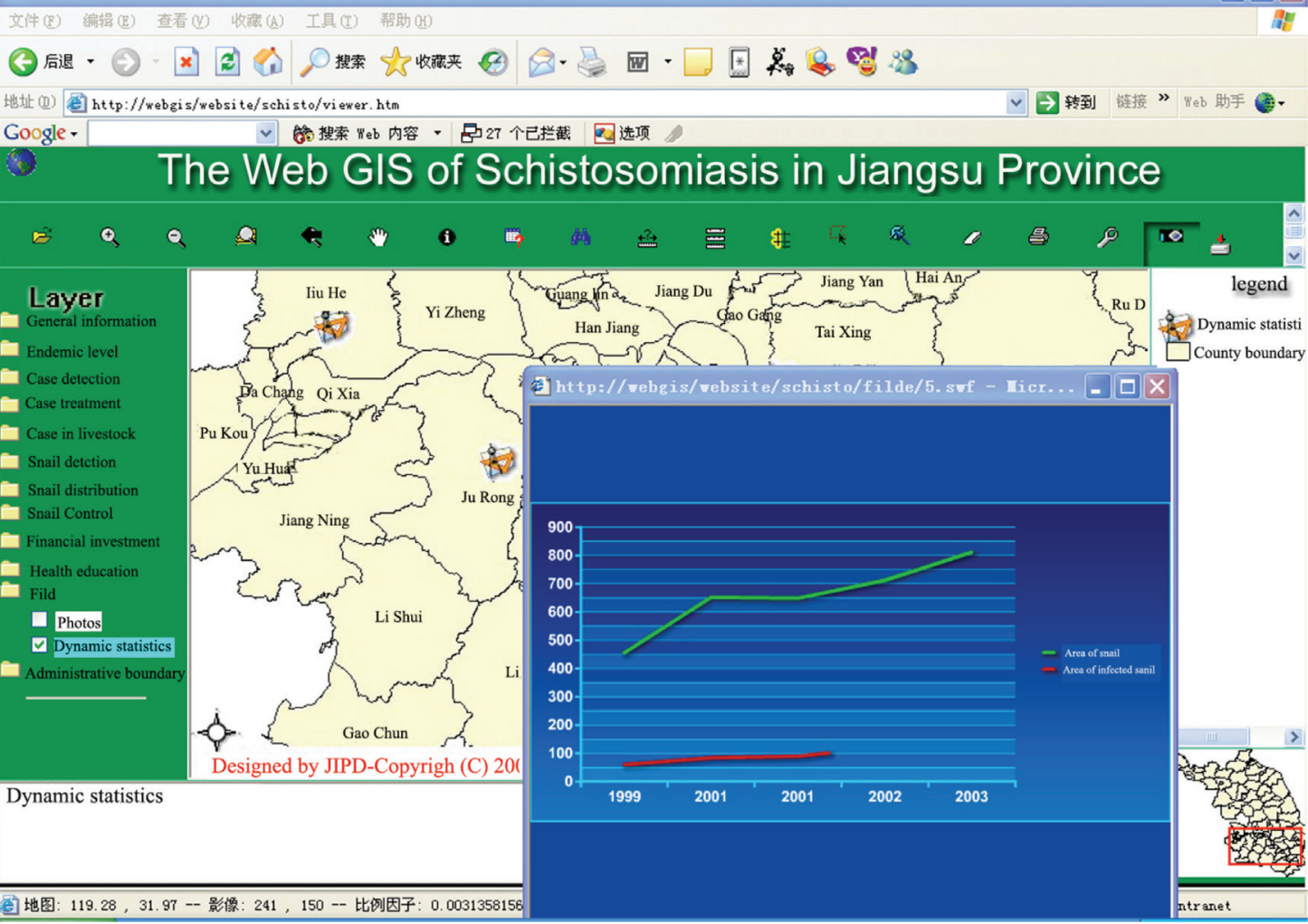

Fig. 3. The function of dynamic statistic analysis of the real-time platform for monitoring schistosomiasis transmission in Jiangsu province, China. 
provided by searches using Google Earth ${ }^{\circledR}$, but also collected through field surveys. Furthermore, it includes advanced risk forecasts based on data stored in the server processed by spatiotemporal modelling, e.g. the WinBugs programme (http://www.mrcbsu.cam.ac.uk/bugs/).

The client interface is similar to the Windows Explorer, i.e. the top and right areas present the tools and the different layers. The tools make it possible to access the main functions of the platform, including directions to open and access the database remotely. The map tools enable effects such as "zooming", "panning", "map tips", "legends" and include advanced analyses including queries, buffer selection, and field information that can show natural still views as well as videos. Management tools for the database are also provided, e.g. system settings along with facilities for downloading, uploading and printing. The layers, which can be enlarged and reduced, include information such as:

(i) current endemic status of schistosomiasis;

(ii) prevalence in humans and livestock;

(iii) snail distribution, snail density and percentage of infected snails;

(iv) control measures implemented;

(v) environmental settings; and

(vi) high-risk regions.

Users can request maps by e-mail or download them in KML format for importation into GIS software packages. They can also get information about historic endemic situations, field information in the form of pictures or videos by pointing (with the mouse) at the geographical areas of interest. Printer-friendly, realtime annotated maps can be generated and printed. The real-time, monitoring release platform was successfully tested and shown to work well with all the commonly used browsers, e.g. Microsoft Internet Explorer, Mozilla Firefox, Google Chrome, Apple Safari and Opera. The platform uses common industry standards, widely accepted data descriptions and communication protocols to facilitate interoperability and portability permitting communication and data transfer between units running on different platforms and using different technologies, e.g. the different versions of Internet Explorer and Firefox as well as the Linux operating systems and MacOS. Before long, the system is planned to be installed on a computer connected with large-scale servers, making it possible for any user with access to the Internet to access the system.

When the WebGIS platform was tested through the local area network at JIPD, the progress of the schistosomiasis control programme could be visualized from the historical point of view as well as in real time, highlighting the decrease of the risk for schistosomiasis in many areas that were considered to be at high-risk as recently as in 2008 and 2009, including clusters of high risk along the Yangtze River in 2008. We were also able to show that decision-makers can easily find the key control areas of schistosomiasis, including the resources available for disease control. The local government of Dantu county, situated along the Yangtze River upstream from Wuxi, tried out the system with immediate results. When consulting the risk maps with all transmission areas pinpointed, including snail densities and the local situation in terms of prevalence, the local action programme could be adapted to the situation at hand without delay (Dai et al., 2011)

\section{Discussion}

New mapping technologies and Google's decision to document the whole world in pictures, including providing free access to satellite imagery, has lead to increased interest worldwide, including public health applications (Chang et al., 2009; Kamadjeu, 2009). Google Earth ${ }^{\circledR}$ has already generated basic, infrastructural data in GIS format (roads, villages, streets, city blocks, schools, health clinics, etc.) and major environmental features (rivers, lakes, etc.) for many regions of the world. Although we are still far from the situation when KML files with infrastructures mapped for the whole world have become available, the regions where such information is currently lacking, or is unreliable, are continously retreating.

It was a priority to establish a platform of components that could be bundled together with relative ease without having to write specific computer programmes. The database of the platform was designed with consideration to the environmental parameters influencing the prevalence in humans, reservoir animals and the intermediate snail host as well as the risk for outbreaks due to the continuous population flux between rural and urban areas, the importance of which for transmission of various diseases has been pointed out by many authors (Engels et al., 2002; Taylor, 2008; Wang et al., 2008).

The platform was designed to provide analyses issuing from remotely sensed data and GIS applications. Active control programmes are constantly generating updated information and the platform should be capable of following and incorporating these developments in real time, the necessity of which has been pointed out many times (Zhou et al., 2007; Taylor, 2008; 
Wang et al., 2008, 2009). Ongoing field operations require constant guidance and the platform must be ready to respond to queries regarding the effectiveness of a particular intervention approach in certain settings or the feasibility of interventions in relation to the resources available, i.e. when and where to intervene and how to identify and locate high-risk areas.

The combination the GIS, remote sensing, and Google Earth technologies offer new opportunities for rapid assessment of endemic areas, provision of reliable estimates of populations at risk, prediction of disease distributions in remote areas that lack baseline data, and guidance of intervention strategies so that scarce resources are allocated in a cost-effective manner (Yang et al., 2005a; Chen et al., 2007; Zhou et al., 2009). The WebGIS approach makes it possible to analyse complex geospatial data and communicate them in a user-friendly graphical format, solving the problem of presenting and explaining the epidemiological situation for decision-makers and the general public on a case by case basis (Theseira, 2002; Croner, 2003; Kamadjeu and Tolentino, 2006; Maclachlan et al., 2007; Syed-Mohamad, 2009). As the technology for WebGIS development becomes more and more readily available, including access to software systems for data management, geographical data visualization through the Internet (e.g. MapServer), geographical data analysis (e.g. ArcGIS), spatial statistics and Internet server management (e.g. Apache) (http://httpd.apache.org/docs/2.0/platform/windows.h $\mathrm{tml})$, general-purpose applications will become more common. The XML-based standard GML is becoming the world standard language for encoding geographic features and geoprocessing service requests (Boulos, 2005). By comparison, many agencies have an extensive WebGIS presence presenting a multitude of digital geospatial data from various areas, e.g. improving hospital bed availability, in community health and bioterrorism surveillance services (Boulos, 2004), and the information of West Nile virus at the United States Geological Survey (USGS) website (http://diseasemaps. usgs.gov/wnv_us_human.html).

The challenge for the real-time surveillance platform is to adapt technologically advanced and costly concepts for disease management to resource-poor environments by developing low-cost tools and solutions (Hrster and Wilbois, 2007; Choo, 2009). Though satellite imagery has been available for over half a century, its broader use was limited until the Landsat programme was initiated in 1972. Due to cost and quality issues, however, the satellite technology has remained in the industrial world and spread only slowly to the developing countries. However, the last few years have seen this 'monopoly' give way to a more global use, at least with regard to the satellite imagery produced. The Web-based interactive platform for schistosomiasis control discussed here is the first of its kind in P.R. China.

\section{Conclusions}

A WebGIS platform, utilizing geo-referenced satellite imagery, freely accessible at the Google Earth ${ }^{\circledR}$ website has been developed for the administration of control programmes of endemic diseases. The useful information available includes risk factors and regions, and target populations at the highest risk for severe disability. The platform can readily be applied to guide schistosomiasis control interventions and disease surveillance in any endemic area and, although it was specifically developed for schistosomiasis, it is a general-purpose application that can be adapted for the surveillance of any disease.

The combination of various technologies into the WebGIS format strengthens overall public health capacity and facilitate decision support system approaches for prevention and control of diseases in resource-poor environments. This platform enables real-time surveillance and risk monitoring with users quickly and intuitively locating any early high-risk area, retrieving detailed performance information and decision makers given the possibility of prompt action.

\section{Acknowledgements}

This work received financial support from the National Natural Science Foundation of P.R. China (grant no. 81101275), Natural Science Foundation of Jiangsu province (BS2010153), Project of Public Health Department of Jiangsu province (grant no. RC2011094), the Chinese Important Scientific Research Project on Infectious Diseases (grant no. 2008ZX10004-011), the UNICEF/UNDP/World Bank/WHO Special Programme for Research and Training in Tropical Diseases (TDR) (A70530), National S \& T Supporting Project (2007BAC03A02), and Shanghai $S$ \& $T$ Project (11XD1405400). The authors would like to acknowledge the staff at many centres for disease control in Jiangsu province for schistosomiasis control for their kind collaboration and making the field data available.

\section{References}

Bergquist R, 2011. New tools for epidemiology: a space odyssey. Mem Inst Oswaldo Cruz 106, 892-900. 
Boulos MN, 2004. Towards evidence-based, GIS-driven national spatial health information infrastructure and surveillance services in the United Kingdom. Int J Health Geogr 3, 1.

Boulos MN, 2005. Web GIS in practice III: creating a simple interactive map of England's Strategic Health Authorities using Google Maps API, Google Earth KML, and MSN Virtual Earth Map Control. Int J Health Geogr 4, 22.

Chang AY, Parrales ME, Jimenez J, Sobieszczyk ME, Hammer SM, Copenhaver DJ, Kulkarni RP, 2009. Combining Google Earth and GIS mapping technologies in a dengue surveillance system for developing countries. Int J Health Geogr 8, 49.

Chen Z, Zhou XN, Yang K, Wang XH, Yao ZQ, Wang TP, Yang GJ, Yang YJ, Zhang SQ, Wang J, Jia TW, Wu XH, 2007. Strategy formulation for schistosomiasis japonica control in different environmental settings supported by spatial analysis: a case study from China. Geospat Health 2, 223-231.

Choo CW, 2009. Information use and early warning effectiveness: perspectives and prospects. JASIS 60, 1071-1082.

Conroy GC, Anemone RL, Van Regenmorter J, Addison A, 2008. Google Earth, GIS, and the Great Divide: a new and simple method for sharing paleontological data. J Hum Evol 55, 751-755.

Cringoli G, Rinaldi L, Veneziano V, Musella V, 2005. Disease mapping and risk assessment in veterinary parasitology: some case studies. Parassitologia 47, 9-25.

Croner CM, 2003. Public health, GIS, and the Internet. Annu Rev Public Health 24, 57-82.

Dai M, Shen XH, Li YF, Chen XP, Zhang LH, Wang L, Hong QB, Yang GJ, 2011. Surveillance of schistosomiasis in a national surveillance site of Dantu district, Zhenjiang city, 2005-2009. Chin J Schisto Control 23, 619-641 (in Chinese).

Engels D, Chitsulo L, Montresor A, Savioli L, 2002. The global epidemiological situation of schistosomiasis and new approaches to control and research. Acta Trop 82, 139-146.

Harrus S, Baneth G, 2005. Drivers for the emergence and reemergence of vector-borne protozoal and bacterial diseases. Int J Parasitol 35, 1309-1318.

Hendrickx G, Biesemans J, de Deken R, 2004. The use of GIS in veterinary parasitology. In Durr P \& Gatrell A (eds) GIS and spatial analysis in veterinary science. Wallingford, CABI Publishing, pp. 145-176.

Hong QB, Yang K, Huang YX, Sun LP, Yang GJ, Gao Y, Zhang LH, Zhou M, Steinmann P, Liang YS, 2011. Effectiveness of a comprehensive schistosomiasis japonica control program in Jiangsu province, China, from 2005 to 2008. Acta Trop 120, S151-157.

Hrster W, Wilbois T, 2007. Early Warning and Risk Management - an Interdisciplinary Approach. Information Technologies in Environmental Engineering - Third International ICSC Symposium 2007, 343-356.

Jia XM, Sriplung H, Chongsuvivatwong V, Geater A, 2009. Sensitivity of pooled serum testing for screening antibody of schistosomiasis japonica by IHA in a mountainous area of Yunnan, China. Parasitology 136, 267-272.

Kamadjeu R, 2009. Tracking the polio virus down the Congo River: a case study on the use of Google Earth in public health planning and mapping. Int J Health Geogr 8, 4.

Kamadjeu R, Tolentino H, 2006. Web-based public health geographic information systems for resources-constrained environment using scalable vector graphics technology: a proof of concept applied to the expanded program on immunization data. Int J Health Geogr 5, 24.

Kamel Boulos MN, 2004. Web GIS in practice: an interactive geographical interface to English Primary Care Trust performance ratings for 2003 and 2004. Int J Health Geogr 3, 16.

Lefer TB, Anderson MR, Fornari A, Lambert A, Fletcher J, Baquero M, 2008. Using Google Earth as an innovative tool for community mapping. Public Health Rep 123, 474-480.

Lillesand TM, Kiefer RW, 1987. Remote Sensing and Image Interpretation, John Wiley and Sons Inc, pp. 708.

Lozano-Fuentes S, Elizondo-Quiroga D, Farfan-Ale JA, LoronoPino MA, Garcia-Rejon J, Gomez-Carro S, Lira-Zumbardo V, Najera-Vazquez R, Fernandez-Salas I, Calderon-Martinez J, Dominguez-Galera M, Mis-Avila P, Morris N, Coleman M, Moore CG, Beaty BJ, Eisen L, 2008. Use of Google Earth to strengthen public health capacity and facilitate management of vector-borne diseases in resource-poor environments. Bull World Health Organ 86, 718-725.

Maclachlan JC, Jerrett M, Abernathy T, Sears M, Bunch MJ, 2007. Mapping health on the Internet: a new tool for environmental justice and public health research. Health Place 13, 72-86.

Malone JB, Yang GJ, Leonardo L, Zhou XN, 2010. Implementing a geospatial health data infrastructure for control of Asian schistosomiasis in the People's Republic of China and the Philippines. Adv Parasitol 73, 71-100.

Otranto D, Capelli G, Genchi C, 2009. Changing distribution patterns of canine vector borne diseases in Italy: leishmaniosis vs. dirofilariosis. Parasit Vectors 2, S2.

Ptochos D, Panopoulos D, Metaxiotis K, Askounis D, 2004. Using internet GIS technology for early warning, response and controlling the quality of the public health sector. Int J Electron Health 1, 78-102.

Rinaldi L, Musella V, Biggeri A, Cringoli G, 2006. New insights into the application of geographical information systems and remote sensing in veterinary parasitology. Geospat Health 1, 33-47.

Rogers DJ, Randolph SE, 2006. Climate change and vectorborne diseases. Adv Parasitol 62, 345-381.

Spear RC, Zhong B, Mao Y, Hubbard A, Birkner M, Remais J, Qiu D, 2004. Spatial and temporal variability in schistosome cercarial density detected by mouse bioassays in village irrigation ditches in Sichuan, China. Am J Trop Med Hyg 71, 554557. 
Stensgaard AS, Saarnak CF, Utzinger J, Vounatsou P, Simoonga C, Mushinge G, Rahbek C, Mohlenberg F, Kristensen TK, 2009. Virtual globes and geospatial health: the potential of new tools in the management and control of vector-borne diseases. Geospat Health 3, 127-141.

Sun LP, Liang YS, Huang YX, Hong QB, Yang G, Zhang LH, Gao Y, Xie CY, 2008. Evaluation on effect of infection control of schistosomiasis in Jiangsu province. Chin J Schisto Control 20, 13-17.

Sun LP, Liang YS, Tian ZX, Dai JR, Hong QB, Gao Y, Huang YX, Yang GJ, Yang K, Wang W, Li W, Zhou XN, 2009. Surveillance and forecast system of schistosomiasis in Jiangsu province. II. Establishment of real-time operation and expression platform based on Google Earth. Chin J Schisto Control 21, 368-372.

Sun LP, Liang YS, Wu HH, Tian ZX, Dai JR, Yang K, Hong QB, Zhou XN, Yang GJ, 2011. A Google Earth-based surveillance system for schistosomiasis japonica implemented in the lower reaches of the Yangtze River, China. Parasit Vectors 4, 223.

Syed-Mohamad SM, 2009. Development and implementation of a web-based system to study children with malnutrition. Comput Methods Programs Biomed 93, 83-92.

Taylor M, 2008. Global trends in schistosomiasis control. Bull World Health Organ 86, 738.

Theseira M, 2002. Using Internet GIS technology for sharing health and health related data for the West Midlands region. Health Place 8, 37-46.

Wang LD, Chen HG, Guo JG, Zeng XJ, Hong XL, Xiong JJ, Wu XH, Wang XH, Wang LY, Xia G, Hao Y, Chin DP, Zhou XN, 2009. A strategy to control transmission of Schistosoma japonicum in China. N Engl J Med 360, 121-128.

Wang LD, Utzinger J, Zhou XN, 2008. Schistosomiasis control: experiences and lessons from China. Lancet 372, 1793-1795.

Yang GJ, Vounatsou P, Tanner M, Zhou XN, Utzinger J, 2006. Remote sensing for predicting potential habitats of Oncomelania hupensis in Hongze, Baima and Gaoyou lakes in Jiangsu province, China. Geospat Health 1, 85-92.

Yang GJ, Vounatsou P, Zhou XN, Utzinger J, Tanner M, 2005a. A review of geographic information system and remote sensing with applications to the epidemiology and control of schistosomiasis in China. Acta Trop 96, 117-129.

Yang K, Liang YS, Yang GJ, Hang YX, Hong QB, Zhu YC, 2005b. Research and design of Internet geographic information system on schistosomiasis. Chin J Schisto Control 17, 365-370.
Yang K, Wang XH, Yang GJ, Wu XH, Qi YL, Li HJ, Zhou XN, 2008. An integrated approach to identify distribution of Oncomelania hupensis, the intermediate host of Schistosoma japonicum, in a mountainous region in China. Int J Parasitol 38, 1007-1016.

Yang K, Zhou XN, Wu XH, Steinmann P, Wang XH, Yang GJ, Utzinger J, Li HJ, 2009. Landscape pattern analysis and Bayesian modeling for predicting Oncomelania hupensis distribution in Eryuan county, People's Republic of China. Am J Trop Med Hyg 81, 416-423.

Zhou XN, Bergquist R, Leonardo L, Yang GJ, Yang K, Sudomo M, Olveda R, 2010. Schistosomiasis japonica: control and research needs. Adv Parasitol 72, 145-178.

Zhou XN, Guo JG, Wu XH, Jiang QW, Zheng J, Dang H, Wang XH, Xu J, Zhu HQ, Wu GL, Li YS, Xu XJ, Chen HG, Wang TP, Zhu YC, Qiu DC, Dong XQ, Zhao GM, Zhang SJ, Zhao NQ, Xia G, Wang LY, Zhang SQ, Lin DD, Chen MG, Hao Y, 2007. Epidemiology of schistosomiasis in the People's Republic of China, 2004. Emerg Infect Dis 13, 1470-1476.

Zhou XN, Lv S, Yang GJ, Kristensen TK, Bergquist NR, Utzinger J, Malone JB, 2009. Spatial epidemiology in zoonotic parasitic diseases: insights gained at the $1^{\text {st }}$ International Symposium on Geospatial Health in Lijiang, China, 2007. Parasit Vectors 2, 10.

Zhou XN, Malone JB, Kristensen TK, Bergquist NR, 2001. Application of geographic information systems and remote sensing to schistosomiasis control in China. Acta Trop 79, 97106.

Zhou XN, Wang LY, Chen MG, Wu XH, Jiang QW, Chen XY, Zheng J, Utzinger J, 2005. The public health significance and control of schistosomiasis in China-then and now. Acta Trop 96, 97-105.

Zhou XN, Yang GJ, Yang K, Wang XH, Hong QB, Sun LP, Malone JB, Kristensen TK, Bergquist NR, Utzinger J, 2008a. Potential impact of climate change on schistosomiasis transmission in China. Am J Trop Med Hyg 78, 188-194.

Zhou YB, Yang MX, Tao P, Jiang QL, Zhao GM, Wei JG, Jiang QW, 2008b. A longitudinal study of comparison of the KatoKatz technique and indirect hemagglutination assay (IHA) for the detection of schistosomiasis japonica in China, 20012006. Acta Trop 107, 251-254.

Zhu YC, Socheat D, Bounlu K, Liang YS, Sinuon M, Insisiengmay S, He W, Xu M, Shi WZ, Bergquist R, 2005. Application of dipstick dye immunoassay (DDIA) kit for the diagnosis of schistosomiasis mekongi. Acta Trop 96, 137-141. 